\title{
Cuidados paliativos em pacientes oncológicos com covid-19
}

\author{
Palliative care in cancer patients with covid-19
}

\section{Cuidados paliativos en pacientes con cáncer con covid-19}

Maria Paula Santana Marques ${ }^{1 *}$, Danilo Rocha Santos Caracas ${ }^{1}$, Tarsis Oliveira Haum ${ }^{3}$, Leonardo Schettini Magalhães Dias ${ }^{1}$, Emmilly Sabrina Mendes de Brito Alves ${ }^{3}$, Rege Farias Oliveira ${ }^{1}$, Bruna Abaliac Azevedo ${ }^{1}$, Adriely Cristina Coronel Ribeiro ${ }^{3}$, Ricardo Rocha Gerbassi ${ }^{2}$, Fillipe Pinheiro Dantas ${ }^{1}$.

\section{RESUMO}

Objetivo: Compreender os cuidados paliativos em pacientes oncológicos acometidos pelo Covid-19. Revisão bibliográfica: $O$ câncer é o crescimento desordenado maligno de células que invadem os tecidos e órgãos, podendo espalhar-se para outras regiões do corpo. Com isso, o próprio câncer ao longo do seu tratamento torna os pacientes mais suscetíveis às infecções, devido ao enfraquecimento da resposta imunológica o organismo fica mais vulnerável à exposição às bactérias e vírus. Pacientes com câncer estão dentro de uma população mais vulnerável e vários fatores podem influenciar na susceptibilidade ao COVID-19, incluindo a idade, o tipo e o estágio do câncer, a sua localização, os efeitos colaterais das escolhas terapêuticas, as visitas frequentes ao sistema de saúde e a prevalência do vírus na região. Com isso, impactando principalmente na qualidade de vida do paciente oncológico e na promoção dos cuidados paliativos, por afetar na manutenção na qualidade de vida e bem-estar. Considerações finais: Dessa forma, torna-se necessário mais estudos sobre os cuidados paliativos em pacientes oncológicos com Covid-19, visando o alívio do sofrimento e a promoção de qualidade de vida.

Palavras-chave: Cuidados paliativos, Câncer, Pandemia, Covid-19.

\begin{abstract}
Objective: To understand palliative care in cancer patients affected by Covid-19. Bibliographic review: Cancer is the disordered, malignant growth of cells that invade tissues and organs and can spread to other regions of the body. With this, the cancer itself during its treatment makes patients more susceptible to infections, due to the weakening of the immune response, the body is more vulnerable to exposure to bacteria and viruses. Cancer patients are within a more vulnerable population and several factors can influence susceptibility to COVID-19, including age, type and stage of cancer, its location, side effects of therapeutic choices, frequent visits to the system and the prevalence of the virus in the region. Thus, mainly impacting the quality of life of cancer patients and the promotion of palliative care, as it affects the maintenance of quality of life and well-being. Final considerations: Thus, further studies are needed on palliative care in cancer patients with Covid-19, aiming to alleviate suffering and promote quality of life.
\end{abstract}

Keywords: Palliative care, Cancer, Pandemic, Covid-19.

\section{RESUMEN}

Objetivo: Comprender los cuidados paliativos en pacientes oncológicos afectados por Covid-19. Revisión bibliográfica: El cáncer es el crecimiento desordenado y maligno de células que invaden tejidos y órganos y pueden diseminarse a otras regiones del cuerpo. Con esto, el cáncer en sí durante su tratamiento hace que los pacientes sean más susceptibles a las infecciones, debido al debilitamiento de la respuesta inmune, el cuerpo es más vulnerable a la exposición a bacterias y virus. Los pacientes con cáncer se encuentran dentro de una población más vulnerable y varios factores pueden influir en la susceptibilidad al COVID-19, incluida la edad, el tipo y estadio del cáncer, su ubicación, los efectos secundarios de las opciones terapéuticas, las

\footnotetext{
${ }^{1}$ Faculdade Santo Agostinho (FASA), Vitória da Conquista - BA.

*E-mail: mariapaulasantanamarques@gmail.com

2 Universidade Federal do Acre (UFAC), Rio Branco - AC.

${ }^{3}$ Campus Faculdade de Guanambi (UniFG), Guanambi - BA.
} 
visitas frecuentes al sistema de salud y la prevalencia del virus. en la región. Así, repercute principalmente en la calidad de vida de los pacientes oncológicos y en la promoción de los cuidados paliativos, ya que incide en el mantenimiento de la calidad de vida y el bienestar. Consideraciones finales: Por lo tanto, se necesitan más estudios sobre cuidados paliativos en pacientes con cáncer con Covid-19, con el objetivo de aliviar el sufrimiento y promover la calidad de vida.

Palabras clave: Cuidados paliativos, Cáncer, Pandemia, Covid-19.

\section{INTRODUÇÃO}

O surgimento da pandemia do coronavírus 2019 (covid-19) causou uma emergência global na saúde pública. O vírus foi detectado pela primeira vez na China e se espalhou por centenas de países (AL-SHAMSI $\mathrm{HO}$, et al., 2020). A estrutura do vírus SARS-Cov-2 é um vírus de RNA de fita simples, envelopado e faz parte da família Coronaviridae (AHMED W, et al., 2020).

A doença pelo covid-19 é uma infecção viral, altamente contagiosa de caráter inflamatório, em que a maioria dos pacientes apresenta de forma leve ou moderada da doença, e aproximadamente $15 \%$ têm a forma grave que requer oxigênio e apenas $5 \%$ dos casos evoluem de forma crítica com complicações como insuficiência respiratória, falência respiratória aguda, choque séptico ou falência múltipla de órgãos (SHARPLESS NE, 2020).

O modo de transmissão do covid-19 é a inalação de aerossóis infecciosos, o período de incubação é de aproximadamente 3 a 14 dias e os sintomas são: febre, tosse, mialgia, dispneia com ou sem diarreia (KANNAN SPAS, et al., 2020). Pelo alto poder contagioso, a doença por covid-19 se tornou uma pandemia desde março de 2020, afetando gravemente a sociedade devido ao seu tempo de incubação. Existem algumas condições de saúde, como Doença Pulmonar Obstrutiva Crônica (DPOC), Hipertensão Arterial Sistêmica (HAS), doenças cardiovasculares e Diabetes Mellitus (DM), que podem aumentar a susceptibilidade ao covid-19 (STERNBERG C, et al., 2020).

Assim, é pertinente considerar o câncer ativo como um dos fatores que podem levar a susceptibilidade devido ao estado de imunossupressão do paciente oncológico (HAN HJ, 2021). O câncer pode ser caracterizado pelo crescimento desorganizado e rápido de células anormais que necessitam de intervenção terapêutica o mais rápido possível, com o objetivo de não deixar o câncer progredir. Além disso, é importante oferecer ao paciente um tratamento que cause menos danos e sequelas ao paciente (PENG L, et al., 2020).

Em pacientes com algumas doenças progressivas incuráveis, como alguns tipos de câncer, é utilizado o tratamento paliativo. Tratamentos paliativos para o câncer, incluindo radioterapia e quimioterapia, desempenham um papel importante na melhoria da qualidade de vida em pacientes com câncer avançado, controlando sintomas e alívio da dor (GHANDOURH WA, 2016). Os cuidados paliativos começam quando o câncer é diagnosticado como incurável e é fornecido por prestadores de cuidados primários ou por profissionais especializados em cuidados paliativos (SIMON ST, et al., 2020).

Além disso, os pacientes com câncer estão em maior risco de adquirir a infecção por COVID-19 e maior probabilidade de ter morbidade e mortalidade mais elevadas do que a população em geral. Inclusive, os fumantes estão em maior risco de vários tipos de câncer, especialmente o câncer de pulmão, com isso tabagismo e câncer de pulmão exacerbam os resultados adversos desses pacientes com covid-19. Ademais, tumores hematológicos e sólidos podem aumentar o risco de várias infecções devido a neutropenia, linfopenia, defeitos humorais e administração de citotóxicos ou de terapias imunossupressoras (MARCHAND L, 2014).

A neutropenia, por exemplo, significa um nível muito baixo de neutrófilos, um tipo de glóbulos brancos que ajuda no combate a infecção, destruindo fungos e bactérias. Assim, pacientes com neutropenia apresentam maior risco de desenvolver infecções graves. Dessa forma, em pacientes com câncer, a função do sistema imunológico pode ser suprimida ou hiper ativada, devido a própria doença ou terapias usadas para tratá-lo. Esta supressão imunológica pode ser um dos fatores implicados no aumento de risco de infecção por covid19 em pacientes com câncer (AHMED W, et al., 2020). 
Em preparação para maior cuidado e utilização de recursos durante a pandemia COVID-19, as estratégias devem ser implementadas para minimizar a interrupção do tratamento do câncer, particularmente em pacientes sendo tratados com intenção curativa. O diagnóstico e o tratamento dos pacientes oncológicos não devem ser comprometidos durante a pandemia de uma doença infecciosa, e por isso o manejo de tais pacientes deve ser com os melhores recursos disponíveis. A necessidade de qualquer procedimento intervencionista deve ser equilibrada contra o risco aumento de contrair o covid-19 e deve ser avaliado de forma que aborda a urgência do procedimento e o efeito sobre o resultado do paciente se o procedimento for avaliado (AL-SHAMSI HO, et al., 2020).

Esse constructo teve como objetivo compreender os cuidados paliativos em pacientes oncológicos acometidos pelo Covid-19.

\section{REVISÃO BIBLIOGRÁFICA}

O diagnóstico precoce do câncer pode levar a um bom prognostico e uma alta taxa de cura, nessa perspectiva o manejo do paciente com câncer é particularmente complexo e deve se equilibrar o risco de um diagnóstico de câncer retardado ou tratamento potencialmente curativo contra o risco óbvio de covid-19 (DENYS A, et al., 2020; SUN Y, et al., 2017).

O recente surto da pandemia covid-19 afetou profundamente e impactou as organizações de saúde em todo o mundo. Novos casos de coronavírus 2019 (covid-19), também conhecida como síndrome respiratória aguda grave coronavírus 2 (SARS-CoV-2), persiste por todo o mundo após a declaração de uma pandemia pela Organização Mundial de Saúde (OMS) (KANNAN SPAS, et al., 2020).

Os sintomas incluem principalmente febre, tosse, distúrbios da respiração, perda olfativa e do paladar temporariamente. Apesar de se manifestar de forma assintomática, é esperado que $20 \%$ dos casos evoluem para formas mais graves da doença, especialmente em indivíduos que possuem fatores de risco, como Hipertensão Arterial Sistêmica, Diabetes Mellitus e neoplasias, idade igual ou superior a 60 anos, transplantados e fumantes (STERNBERG C, et al., 2020).

A estrutura do vírus SARS-CoV-2 é de RNA de fita simples e envelopado. O envelope é composto do CoV2 é composto pela glicoproteína Matrix (M) e proteína envelope (E) e é revestida pela proteína Spike (S) que desempenha um papel fundamental na ligação viral às células hospedeiras e internalização: foi demonstrado que ela se liga ao receptor da superfície a Enzima Conversora da Angiotensina (ACE2) das células epiteliais na árvore respiratória e logo após é clivada por uma serina protease hospedeira, Transmembrane Serina Protease 2 (TMPRSS2), para ser internalizada (CAO X, 2020).

A ACE2, que é ligada à membrana e desempenham um papel fundamental na manutenção da pressão arterial. A expressão do ACE2 no tecido pulmonar, onde se pensa que desempenha um papel fundamental na infecção pelo SARS-CoV-2, tem sido sugerido ser aumentado com a idade. Uma vez que os pacientes com câncer são em média mais velhos que o geral, foi proposto que este seja um dos mecanismos que resultam em efeitos adversos em pacientes mais velhos (CAO X, 2020).

As evidências, sugerem que a resposta imune a SARS-CoV-2 pode desempenhar papéis diferentes. Pacientes que têm certas formas de imunidade primárias ou secundárias suprimidas por algum mecanismo, parecem estar em risco aumentado quando desenvolvem covid-19. Existe também a evidência que sugere que os pacientes que foram infectados pelo covid-19 e evoluíram para forma grave, apresentaram uma tempestade de citocinas que parecem ter níveis aumentados, com a interleucina (IL-6), demonstrando a importância de um sistema imunológico intacto para uma resposta satisfatória à infecção por SARS-CoV-2 (AHMED W, et al., 2020).

A atual pandemia alterou de maneira significante o fluxo de cuidados nos serviços de saúde no mundo todo. No entanto, mesmo durante esse período crítico, os pacientes com outras doenças, como câncer, precisam ser tratados adequadamente. A mortalidade devido ao SARS-CoV-2 é maior em pacientes idosos e aqueles com outras comorbidades ativas, incluindo o câncer (ZHANG H, et al., 2020). 
Com isso, o próprio câncer ao longo do seu tratamento torna os pacientes mais suscetíveis às pneumonias, e devido ao enfraquecimento da resposta imunológica o organismo fica mais vulnerável à exposição às bactérias e vírus. Pacientes com câncer de pulmão, por exemplo, geralmente têm o pulmão comprometido. Eles podem estar em maior risco de formas graves da infecção do COVID-19, devido a diminuição da função pulmonar. Assim, a propagação da epidemia de COVID- 19 representou grandes desafios para a prática clínica de oncologistas, especialmente para a terapia integrativa do câncer (YANG G, et al., 2020).

Na terapia sistêmica oncológica, as decisões, principalmente durante a pandemia do covid-19 devem ser orientadas considerando cuidados e características individuais do sistema imunológico. O diálogo e as decisões compartilhadas entre o médico e o paciente, devem se concentrar nos benefícios da terapia proposta se contrapondo com o risco de o paciente contrair COVID-19 enquanto o tratamento é direcionado ao câncer. As terapias direcionadas ao câncer que tem potencial curativo, de preferência não devem ser atrasadas. Nesses casos, a telemedicina pode ser considerada para apoiar os pacientes remotamente para reduzir as visitas no hospital durante uma pandemia infecciosa. A telemedicina demonstrou melhorar o acesso aos cuidados e diminuir os custos da saúde (AHMED W, et al., 2020).

As principais limitações da telemedicina incluem os treinamentos em ferramentas digitais e limitações do exame físico. Pacientes que estão em risco ou contraíram o covid-19, devem ser vistos e tratados em ambientes separados. Além disso, e não menos importante, dentro do tratamento dos pacientes oncológicos em terapias paliativas ou não, inclui a prevenção e educação em saúde, sobre a importância da higiene de mãos, medidas para o controle da infecção e os sinais e sintomas do covid-19. Dentro desse contexto, também deve ser considerado o adiamento de cirurgias eletivas ou quimioterapia para pacientes com câncer com baixo risco de progressão (MADARIAGA A, et al., 2020).

As escolhas feitas no momento de qualquer tratamento, assim como em toda área da medicina, devem ser destinadas principalmente a melhorar o paciente e proporcionar uma melhor qualidade de vida. Essa conduta não seria diferente com os pacientes oncológicos em cuidados paliativos. Que tem como objetivo promover bem-estar enquanto o paciente estiver sendo assistido (MOUNSEY I, et al., 2019).

Essa realidade inserida no contexto atual se torna ainda mais difícil. A doença pela nova cepa do coronavírus, foi classificada pela OMS como pandemia, o que dificultou ainda mais o âmbito hospitalar do mundo inteiro. Uma das principais características do SARS-CoV-2 é o alto nível de contagiosidade. A principal via de transmissão de pessoa para pessoa é através de gotículas geradas por uma tosse ou um espirro de uma pessoa infectada, por exemplo. Essas gotas são espalhadas pelo ar e pode depositar nas membranas da mucosa da boca, do nariz ou dos olhos. Dessa forma, no ambiente médico o risco de transmissão do vírus é potencializado, pela grande circulação diária de pessoas que estão adoecidas, até mesmo contaminadas com o coronavírus (PEIXOTO RD e LOPES MDSM, 2020).

Pacientes com câncer estão dentro de uma população mais vulnerável e vários fatores podem influenciar na susceptibilidade ao COVID-19, incluindo a idade, o tipo e o estágio do câncer, a sua localização, os efeitos colaterais das escolhas terapêuticas, as visitas frequentes ao sistema de saúde e a prevalência do vírus na região. Com isso, impactando principalmente na qualidade de vida do paciente oncológico e na promoção dos cuidados paliativos, por afetar na manutenção na qualidade de vida e bem-estar. A terapia atual do câncer se expandiu para além do tratamento dos tratamentos quimioterápicos tradicionais, bem como imunoterapia, resultando em alguns potenciais tóxicos (RICHARDS M, et al., 2020).

Em comparação com a população que não apresente nenhum tipo de câncer, os pacientes oncológicos apresentam um maior risco de complicações relacionadas ao tromboembolismo, que pode resultar em maior morbimortalidade neste grupo. Além disso, esse alto risco de maior complicação de infecção por SARS-CoV2 em pacientes com câncer também é explicada por um serie de mecanismos imunológicos, como o estado imunossupressor que é causado por uma série de tumores e pela substancia citotóxica comumente usadas em terapias (MONTEIRO MR, et al., 2021).

Esses fatores levam uma supressão da resposta imunológica do indivíduo, como por exemplo, através de linfopenia e/ou neutropenia. Essa resposta imunológica prejudicada leva a persistência do vírus, garantindo 
uma continua liberação de citocinas, levando a danos mais graves ao pulmão. Assim, através dessa análise é possível concluir o quanto a infecção contribui para piorar o prognostico geral do paciente com neoplasia quando infectado por SARS-CoV-2 (AMORIM GLDS, et al., 2020; SAMPAIO SGSM, et al., 2021).

Os pacientes oncológicos terminais se beneficiam de uma abordagem paliativa, que compreende atenção e alívio dos sintomas, as necessidades psicossociais e ao apoio do cuidador. Muitas vezes os cuidados paliativos estão ligados a uma trajetória da doença altamente previsíveis, seguido com o tempo de preservação funcional seguido por um declínio terminal. Porém, em vários casos e tipos de câncer, é importante que desde o diagnostico seja aplicado medidas paliativas, a fim de modificar o curso da doença, prevenindo uma piora significativa e assim oferecer ao paciente uma oportunidade de tratar com o mínimo de efeitos colaterais possíveis (DY SM, et al., 2017; ROBILOTTI EV, et al., 2020).

As dificuldades que os pacientes com câncer enfrentam para acessar e oferecer cuidados de saúde aumentou à medida que a crise da pandemia do COVID-19 continua. Com isso, $79 \%$ dos pacientes em tratamento ativo para o câncer tiveram um atraso em seus cuidados de saúde, incluindo $17 \%$ diz respeito a atrasos na quimioterapia, radiação ou terapia hormonal. Atrasos também foram comuns entre pacientes que não estão atualmente em tratamento ativo, como cuidados relacionados ao curso da doença, bem como cuidados regulares/preventivos, incluindo os cuidados paliativos (PESTANA RC, et al., 2020).

Pacientes com câncer em tratamento de fim de vida, sendo apenas paliativo que são acometidos pelo covid-19, foram considerados, de maior variável para o aumento da mortalidade, assim como admissão em Unidade de Terapia Intensiva (UTI) e Ventilação Mecânica (VM). Em relação as características do câncer, embora o câncer de pulmão aumente o risco de morte, paciente com metástase teve um resultado ainda pior em relação as taxas de mortalidade (KAYE EC, et al., 2015).

A fragilidade do paciente oncológico o colocou em um grupo de risco para covid-19, dessa forma faz se necessário o preparo de equipes bem qualificadas, para assim, garantir uma assistência segura, principalmente nos casos dos pacientes que já avançaram para cuidados paliativos em fase terminal, com um maior controle da sintomatologia dessa fase. Nessa circunstância fez se necessário alterações e revisão de protocolos assistências na área da saúde, bem como no setor de cuidados paliativos da oncologia, para que seja priorizado a segurança e o bem do paciente e dos seus familiares (XU Y, et al., 2020).

Outra importante ferramenta que deve ser ressaltada é a comunicação e o trabalho em equipe para que a segurança desses pacientes seja garantida. Apesar das dificuldades, o plano de cuidados deve continuar individualizado e humanizado, porém com o mínimo de exposição ocupacional. Em pacientes isolados, contaminados ou com suspeita de contaminação pelo SARS-CoV-2, o planejamento de cuidados deve ser direcionado a promoção do conforto, equilíbrio físico e emocional. Ademais, o paciente oncológico já possui alguns fatores que os deixam fragilizados, tanto o próprio diagnostico quando o tratamento (KAYE EC, et al., 2015).

Além disso, a saúde mental do paciente deve ser considerada por ser afetada por vários fatores, como psicológicos, espirituais e sociais. Diante desse contexto, é importante que a equipe multidisciplinar responsável por assistir o paciente oncológico em cuidados paliativos afetado pelo covid-19, continue 0 tratamento adequado de forma que minimize ou controle os sintomas tanto do covid-19, quanto da neoplasia, e sempre respeitando as medidas adotadas para controle de disseminação do coronavírus. Os cuidados devem-se garantir o conforto e a dignidade, promovendo qualidade de vida e de morte, mesmo com os obstáculos impostos pela atual pandemia do covid-19 (PESTANA RC, et al., 2020).

Em pacientes terminais, o fim da vida poderia ser definido como aquela em que o indivíduo não tenha dor e sofrimento, ou que os mesmos sejam amenizados por meio de cuidados paliativos, por meio de uma equipe multidisciplinar. Além disso, é importante esses cuidados envolverem empatia, descontração e compreensão por parte da equipe de saúde e familiares (DY SM, et al., 2017; LYU T, et al., 2020).

A dispneia pode estar presente em pacientes com câncer avançado, sendo sempre importante avaliar a causa base. É um sintoma que se associado a infecção pelo covid-19 pode ser ocasionado ou exacerbado 
pelo vírus, visto que o microrganismo é inalatório, há uma maior preferência de alojação pulmonar que causa uma "tempestade inflamatória" acometendo os brônquios que levam aos problemas respiratórios (VELA-RUIZ JM, et al., 2020; DY SM, et al., 2017; LYU T, et al., 2020).

Dessa forma, pode haver uma piora do prognostico do paciente em tratamento, sendo assim, importante garantir o bem-estar do paciente seja ele em curso da doença ou em fase terminal, aliviando os sintomas por meio de cuidados e medicações necessárias. Outro sintoma que também pode ser exacerbado pela presença do coronavírus no organismo do indivíduo, é a fadiga. Sendo este, o sintoma mais comum em pacientes oncológicos em estado avançado (VELA-RUIZ JM, et al., 2020).

A perda do apetite é o segundo sintoma mais comum em pacientes com câncer avançado, o que leva a anorexia. Apetite e ingestão alimentar são fatores importantes para uma boa qualidade para o paciente. Caso não seja alimentado da forma correta e saudável, acarreta uma diminuição nos nutrientes necessários para o organismo, o que pode levar a uma queda de imunidade. Assim, é importante que caso possível haja a ingestão em pequenas quantidades e em intervalos regulares. Caso indicado, utilizar as medicações necessárias (MOUNSEY I, et al., 2018).

A alteração da mucosa oral, pode ser um efeito da radioterapia ou da quimioterapia, o que pode causar uma diminuição da ingestão oral e predispõe a infecções. Pode ser causado, por ansiedade ou por tumoração local, por exemplo. Dessa forma, é importante manter a higiene bucal, controlar a dor ou as infecções com as medicações corretas (LENIHAN D, et al., 2020; HAN HJ, et al., 2021; NASCIMENTO CC, et al., 2020).

Dessa forma, a pandemia do COVID-19 exige uma resposta rápida de profissionais de cuidados oncológicos para fornecer prevenção adequada, orientação, identificação e tratamentos adequados e avaliação de casos críticos, incluindo a decisão de adiar ou interromper o tratamento (SCHRAG D, et al., 2020).

\section{CONSIDERAÇÕES FINAIS}

Indivíduos em terapia anticâncer e infectados pelo covid-19, têm mais chances de evoluir com as formas mais graves da doença. Como o indivíduo apresenta em um estado de imunossupressão causado pelo câncer e pelo tratamento do câncer, os indivíduos oncológicos estão mais susceptíveis às infecções do trato respiratório do que os indivíduos sem neoplasias. Dessa forma, torna-se necessário mais estudos sobre os cuidados paliativos em pacientes oncológicos com covid-19, visando o alívio do sofrimento e a promoção de qualidade de vida.

\section{REFERÊNCIAS}

1. AHMED W, et al. Decomposição do SARS-CoV-2 e do vírus da hepatite murina substituta RNA em águas residuais não tratadas para informar a aplicação em epidemiologia baseada em águas residuais. Pesquisa Ambiental, 2020; 191: 110092.

2. AL-SHAMSI HO, et al. A practical approach to the management of cancer patients during the novel coronavirus disease 2019 (COVID-19) pandemic: an international collaborative group. The oncologist, 2020; 25(6): e936.

3. AMORIM GLDS, et al. Breast oncology and the COVID-19 pandemic: recommendations from the Brazilian Society of Clinical Oncology (SBOC). Brazilian Journal of Oncology, 2020; 16: e20190024.

4. CAO X. COVID-19: immunopathology and its implications for therapy. Nature reviews immunology, 2020; 20(5): 269270, 2020.

5. DENYS A, et al. Oncologia intervencionista na época da pandemia COVID-19: problemas e soluções. Diagnóstico e intervenção de imagem, 2020; 101(6): 347-353.

6. DY SM, et al. Palliative care for cancer survivors. Medical Clinics, 2017; 101(6): 1181-1196.

7. GHANDOURH WA. Palliative care in cancer: managing patients' expectations. Journal of medical radiation sciences, 2016; 63(4): 242-257.

8. HAN HJ, et al. COVID-19 and cancer: From basic mechanisms to vaccine development using nanotechnology. International immunopharmacology, 2021; 90: 107247.

9. KANNAN SPAS, et al. COVID-19 (Novel Coronavírus 2019) - recent trends. European Review for Medical and Pharmacological Sciences, 2020; 24(4): 2006-2011. 
10. KAYE EC, et al. Pediatric palliative care in the community. A Cancer Journal for Clinicians, 2015; 65(4): $315-333$.

11. LENIHAN D, et al. Cardio-oncology care in the era of the coronavirus disease 2019 (COVID-19) pandemic: An International Cardio-Oncology Society (ICOS) statement. A Cancer Journal for Clinicians, 2020; 70(6): 480-504.

12. LYU T, et al. Expert consensus on the procedure of interventional diagnosis and treatment of cancer patients during the COVID-19 epidemic. Journal of Interventional Medicine, 2020; 3(2): 61-64.

13. MADARIAGA A, et al. COVID-19 Testing in Patients with Cancer: Does One Size Fit All? Clinical Cancer Research, 2020; 26(18): 4737-4742.

14. MARCHAND L. Integrative and complementary therapies for patients with advanced cancer. Annals of palliative medicine, 2014; 3(3): 160-171.

15. MONTEIRO MR, et al. Cancer patients diagnosed with COVID-19 infection: a multicenter retrospective cohort of nine Brazilian cancer centers. Brazilian Journal of Oncology, 2021; 1: 1-10.

16. MOUNSEY L, et al. Palliative care for the patient without cancer. Australian journal of general practice, 2019; 47(11): 765-769.

17. NASCIMENTO CC, et al. Desafios e Recomendações à Atenção Oncológica durante a Pandemia da Covid-19. Revista Brasileira de Cancerologia, 2020; 66: e1241.

18. PEIXOTO RD, LOPES MDSM. COVID-19 among cancer patients. What we know so far? Brazilian Journal of Oncology, $2020 ; 16: 1-3$

19. PENG L, et al. Gerenciamento de pacientes com câncer na era COVID-19. The European Journal of Cancer, 2020; 132: $5-7$.

20. PESTANA RC, et al. COVID-19 incidence and outcomes among patients with respiratory symptoms in a cancer center emergency department. Brazilian Journal of Oncology, 2020; 16: 1-5.

21. RICHARDS M, et al. The impact of the COVID-19 pandemic on cancer care. Nature Cancer, 2020; 1(6): $565-567$.

22. ROBILOTTI EV, et al. Determinants of COVID-19 disease severity in patients with cancer. Nature medicine, 2020; 26(8): 1218-1223.

23. SAMPAIO SGSM, et al. Evaluation of the Criteria Adopted to Identify Suspected Cases of COVID-19 in the Emergency Department Service of a Referral Palliative Oncology Care Unit. American Journal of Hospice and Palliative Medicine, 2021; 38(2): 199-203.

24. SCHRAG D, et al. Oncology practice during the COVID-19 pandemic. The Journal of the American Medical Association, 2020; 323(20): 2005-2006.

25. SHARPLESS NE. COVID-19 and cancer. Science, 2020; 368(6497): 1290.

26. SIMON ST, et al. The palliative care of patients with incurable cancer. Deutsches Ärzteblatt International, 2020; 117(7): 108.

27. STERNBERG C, et al. Oncology practice during COVID-19 pandemic: a fast response is the best response. Revista da Associação Médica Brasileira, 2020; 66(3): 338-344.

28. SUN Y, et al. Risk factors and preventions of breast cancer. International journal of biological sciences, 2017; 13(11): 1387.

29. VELA-RUIZ JM, et al. Desafíos en la atención de los pacientes con cáncer durante la pandemia COVID-19. Revista Peruana de Medicina Experimental y Salud Pública, 2020; 37: 580-581.

30. XU, Yan et al. Clinical recommendations on lung cancer management during the COVID-19 pandemic. Thoracic Cancer, 2020; $11(7):$ 2067-2074.

31. YANG G, et al. Challenges and countermeasures of integrative cancer therapy in the epidemic of COVID-19. Integrative cancer therapies, 2020; 19: e1534735420912811.

32. ZHANG H, et al. Treatment and outcome of a patient with lung cancer infected with severe acute respiratory syndrome coronavirus-2. Journal of Thoracic Oncology, 2020; 15(5): 63-64. 